\title{
Pregnancy outcomes in women with repeated implantation failures after intracytoplasmic morphologically selected sperm injection (IMSI)
}

\author{
João Batista A Oliveira ${ }^{1,2,3^{*}}$, Mario Cavagna ${ }^{3}$, Claudia G Petersen ${ }^{1,2,3}$, Ana L Mauri, ${ }^{2,3}$, Fabiana C Massaro ${ }^{2,3}$,
} Liliane FI Silva ${ }^{2,3,4}$, Ricardo LR Baruffi ${ }^{2,3}$ and Jose G Franco Jr ${ }^{1,2,3}$

\begin{abstract}
Background: The purpose of this study was to compare laboratory and clinical outcomes of intracytoplasmic morphologically selected sperm injection (IMSI) and conventional intracytoplasmic sperm injection (ICSI) in couples with repeated implantation failures.

Methods: A total of 200 couples with at least two prior unsuccessful ICSI cycles were enrolled: 100 couples were submitted to IMSI and 100 were submitted to routine ICSI. For IMSI, spermatozoa were selected at 8400X magnification using an inverted microscope equipped with Nomarski (differential interference contrast) optics. For conventional ICSI, spermatozoa were selected at 400x magnification. Clinical outcomes were evaluated between the two groups.

Results: Study patients were comparable in age, number of treatment failures, aetiology of infertility, percentage of normal form assessed by MSOME (motile sperm organelle morphology examination), semen parameters, total number of oocytes collected, number of mature oocytes collected, total number of embryos transferred and number of high-quality embryos transferred. No statistically significant differences between the two groups were observed with regard to rates of fertilisation, implantation and pregnancy/cycle. Although not statistically significant, rates of miscarriage (IMSI:15.3\% vs ICSI:31.7\%), ongoing pregnancy (IMSI:22\% vs ICSI:13\%) and live births (IMSI:21\% vs ICSI:12\%) showed a trend towards better outcomes in the IMSI group. In addition, analysis of subpopulations with or without male factor showed similar results.

Conclusions: Our results suggest that IMSI does not provide a significant improvement in clinical outcome compared to ICSI, at least in couples with repeated implantation failures after conventional ICSI. However, it should be noted that there were clear trends for lower miscarriage rates $(\approx 50 \%$ reduced) and higher rates of ongoing pregnancy and live births (both nearly doubled) within the IMSI group. Further confirmation as well as randomized large-scale trials are needed to confirm the beneficial effects of IMSI in couples with poor reproductive prognoses.
\end{abstract}

\section{Background}

Implantation failure is the major cause negatively influencing the outcome of assisted reproductive technologies (ART), as only two out of every ten embryos successfully implant [1]. The outcome of intracytoplasmic sperm injection (ICSI) has been shown to be positively associated with the morphological state of the sperm [2-7],

\footnotetext{
* Correspondence: joaobatista@crh.com.br

'Department of Gynecology and Obstetrics, Botucatu Medical School Sao

Paulo State University - UNESP, Botucatu, Brazil

Full list of author information is available at the end of the article
}

while early miscarriage rates were negatively associated with nuclear morphology [5-7]. However, repeated failure of conventional IVF has been suggested to be caused by a paternal effect on early embryo development, a hypothesis confirmed using a shared oocyte donation model [8-11].

Bartoov et al. [5,12] developed a method of human spermatozoa evaluation performed in real-time at high magnification called "motile sperm organelle morphology examination" (MSOME). MSOME is performed using an inverted microscope equipped with Normarski

\section{C) Biomed Central}


interference contrast optics, which enables observation at high magnification $(>6000 \times)$ compared to the 200$400 \times$ observed by conventional ICSI [12]. This method led to the development of the intracytoplasmic morphologically selected sperm injection (IMSI) procedure, which is based on sperm normality as defined by MSOME classification and aims to improve conventional ICSI outcomes by focusing on the correlation between abnormalities in sperm morphology observed at high magnification and DNA damage [5,13-20]. Various studies have demonstrated that IMSI significantly improves fertilisation rates [21,22], embryo quality $[5,11,20,21,23]$, the rate of development up to the blastocyst stage $[21,24]$, the rates of implantation and pregnancy after embryo transfer on day 2 or 3 $[5-7,11,13,20,22,23,25,26]$ or in the blastocyst stage $[24,27]$ and the likelihood of having a normal healthy child [28]; IMSI also appears to significantly decrease miscarriage rates [5-7,13,23,26,29]. In fact, prior failures in ICSI cycles constituted an inclusion criterion in several studies employing IMSI $[6,13,24,28,30]$.

To better comprehend the value of IMSI/MSOME, the purpose of this study was to compare laboratory and clinical outcomes of IMSI versus conventional ICSI in couples with repeated ICSI failures.

\section{Materials and methods Study participants}

A total of 200 couples with at least two prior implantation failures were prospectively evaluated. Patients were divided into two groups matched by (female) age:

Group ICSI: 100 patients submitted to conventional ICSI.

Group IMSI: 100 patients submitted to IMSI.

All couples enrolled in the study met the following inclusion criteria: subfertility with a need for assisted reproduction with ejaculated spermatozoa, two or more failed ICSI attempts with embryos of good morphological quality (four identical blastomeres isolated $44 \mathrm{~h}$ after sperm injection with no fragments or multinucleation present [31-33]), a maternal age $\leq 39$ years, a normal karyotype in both partners and negative for uterine defects, ultrasonographic evidence of hydrosalpinx, evidence of low ovarian response in previous treatment cycles (less than four oocytes retrieved), infections, endocrine problems, coagulation defects or thrombophilia and autoimmune defects (including antiphospholipid antibodies). The division between groups was performed as follows: after referral of the patient for IMSI, the subsequent patient with the same characteristics (i.e., least two implantation failures, same age ( \pm 1 year) and fulfilled the inclusion criteria) was referred for ICSI. Written informed consents were obtained from all couples, and this study was approved by an internal institutional review board.

\section{Ovarian stimulation and oocyte recovery culture protocol}

All patients were submitted to the same scheme of controlled ovarian stimulation. First, pituitary downregulation was established with nafarelin acetate at a dose of $400 \mathrm{mg} /$ day $\left(\right.$ Synarel ${ }^{\circledR}$; Pfizer, Brazil) started during the luteal phase of the previous cycle. After 14 days of treatment with the GnRH analogue and after a confirmation of downregulation, the administration of recombinant FSH (Gonal F ${ }^{\circledR}$; Serono Barueri, SP, Brazil) was initiated at a dose of 150-250 IU, depending on patient age, and $75 \mathrm{IU} /$ day of recombinant LH (Luveris ${ }^{\circledR}$, Serono, SP, Brazil) for a period of 7 days. When one or more follicles measuring $\geq 17 \mathrm{~mm}$ were observed, recombinant HCG (Ovidrel ${ }^{\mathbb{B}}$; Serono, Brazil) was administered at a dose of $250 \mu \mathrm{g}$, and oocyte retrieval was scheduled 35-36 hours later.

\section{Semen preparation}

Semen samples were collected in sterile containers by masturbation after a sexual abstinence period of 2 to 5 days. The liquefied, fresh semen samples were prepared using ISolate (Irvine Scientific, Santa Ana, CA, USA) discontinuous concentration gradient. The final pellet was resuspended in $0.2 \mathrm{~mL}$ of modified HTF medium (Irvine Scientific, Santa Ana, CA, USA) supplemented with 10\% human serum albumin (Irvine Scientific, Santa Ana, CA, USA).

Each semen sample was analysed for morphology according to the MSOME criteria [12] and for standard semen quality parameters according to the World Health Organisation[34].

\section{ICSI procedure}

Conventional ICSI was performed using a Nikon Eclipse TE 300 inverted microscope equipped with Narishige 231 D-2 (Narishige, Tokyo, Japan) remote control hydraulic micromanipulators and Narishige IM-9B injectors. Spermatozoa were selected at $400 \times$ magnification using Hoffman modulation contrast following a set of published guidelines.

\section{IMSI procedure}

A $1-\mu \mathrm{L}$ aliquot of sperm cell suspension was transferred to a 5- $\mu \mathrm{L}$ microdroplet of modified HTF medium containing 7\% polyvinylpyrrolidone solution (PVP medium Irvine Scientific, USA). The microdroplet was placed into a sterile glass dish (FluoroDish ${ }^{\mathrm{TM}}$ Word Precision Instrument, USA) under sterile paraffin oil (Ovoil-100, Vitrolife, Goteborg, Sweden). Sperm cells suspended within the microdroplet were placed on a microscope 
stage above an Uplan Apo 100 oil/1.35 objective lens previously covered by a droplet of immersion oil. In this manner, suspended motile sperm cells in the observation droplet could be examined at high magnification through an inverted microscope (Eclipse TE $2000 \mathrm{U}$ Nikon, Japan) equipped with high-powered differential interference contrast optics (DIC/Nomarski). Images were captured using a colour video camera containing effective picture elements (pixels) for high-quality image production and projected onto a colour video monitor. Morphological evaluation was accomplished using a monitor screen, and the total calculated magnification was $8400 \times$ (total magnification: objective magnification $=$ $100 \times$, magnification selector $=1.0 \times$, video coupler magnification $=1.0 \times$, calculated video magnification $=$ $84.50 \times)$.

A spermatozoon used for IMSI was classified as morphologically normal if it exhibited a normal nucleus as well as an acrosome, a post-acrosomal lamina, a neck and a tail, and did not present a cytoplasmic droplet or cytoplasm around the head [12]. The subcellular organelles were morphologically classified based on the presence of specific malformations, which were defined according to the arbitrary descriptive approach reported by Bartoov et al. [12] after studies utilising transmission and scanning electron microscopy. According to transmission electron microscopy estimations $[6,12]$, a morphological normal state for the nucleus was defined by the shape and content of the chromatin. The criterion for normality of nuclear form was a smooth, symmetric and oval configuration. Normal means for length and width were estimated as $4.75 \pm 2.8$ and $3.28 \pm 0.20 \mu \mathrm{m}$ [12], respectively, and the form was classified as abnormal when it presented a variation of 2 standard deviations in one of the axes (length: $\geq 5.31$ or $\leq 4.19 \mu \mathrm{m}$; width: $>3.7$ or $<2.9 \mu \mathrm{m}$ ). For rapid evaluation of nuclear form, a fixed, transparent, celluloid form of a sperm nucleus fitting the criteria was superimposed on the examined cell (chablon construction based on ASTM E 1951-2 [35]). In the same manner, the nuclear form was considered abnormal if extrusion or invagination of the nuclear chromatin mass was detected (regional malformation of nuclear form). Chromatin content was considered abnormal if one or more vacuoles were observed to occupy more than $4 \%$ of the nuclear area (visual evaluation was aided by a celluloid form of a large vacuole superimposed on the examined cell, if necessary). A nucleus was considered normal if both nuclear form and chromatin content were normal.

The same technician performed all sperm selection, and only normal spermatozoa were injected in this study. The time involved in the selection step was 30$120 \mathrm{~min} /$ sample, and at least three spermatozoids were selected for each MII oocyte. After sperm selection, microinjections were carried out in the same manner as in ICSI. Spermatozoids were still motile when captured for final selection.

\section{Oocyte and embryo culture and transfer}

Sperm-injected oocytes, zygotes and embryos from both IMSI and ICSI groups were submitted to the same culture conditions. On day 2, the two/three best-scoring embryos from both ICSI and IMSI groups were transferred. The quality of transferred embryos was similar between the groups.

\section{Statistical analysis}

Data were analysed using InStat version 3.0 (GraphPad Software, San Diego California, USA) on a Macintosh computer (Apple Computer Inc., Cupertino, California, USA). The primary endpoint of the investigation was the ongoing pregnancy rate and secondary endpoints were the rates of fertilisation, clinical pregnancy per cycle, miscarriage and live births. Fertilisation was defined as zygotes containing two pronuclei after ICSI or IMSI. Pregnancy was defined as the presence of a gestational sac with heartbeat visualized by ultrasound 4-6 weeks after embryo transfer. Miscarriage was defined as the termination of the pregnancy up to 20 weeks of gestation. The variables were analysed in relation to the general population as well as the subpopulations with or without male factor. The sample size for the general study was calculated by comparing between two proportions: control (ICSI cycles) and experimental (IMSI cycles). In general, the mean ongoing pregnancy rate was $25 \%$. Thus, a sample size of 100 patients in each group had $80 \%$ power to detect an increase of $20 \%$ with an alpha significance level of 0.05 (two-tailed). When appropriate, Student's $t$-tests, Mann-Whitney tests and chi-square tests were utilised with a significance level of $P<0.05$.

\section{Results}

The characteristics of the study patients (general population) in both the IMSI and ICSI groups were comparable with regard to age, the number of treatment failures, aetiology of infertility, the percentage of normal form assessed by MSOME, semen parameters, the total number of oocytes collected, the number of mature oocytes collected, the total number of embryos transferred and the number of high-quality embryos transferred. No statistically significant differences $(P>0.05)$ were observed between the two groups with regard to rates of fertilisation (IMSI: $65.4 \pm 23.5 \%$; ICSI: $62 \pm 26.5 \%$ ), implantation (IMSI:13.6\%; ICSI:9.8\%) and pregnancy/cycle (IMSI:26\%; ICSI:19\%). Although not statistically significant, rates of miscarriage (IMSI:15.4\%; ICSI:31.6\%), ongoing pregnancy (IMSI:22\%; ICSI:13\%) and live births (IMSI:21\%; 
ICSI:12\%) displayed a clear trend towards better outcomes in the IMSI group. Table 1 summarises the characteristics and outcomes of the general study population.

In the subpopulation of cases with male factor (IMSI: 37 couples; ICSI: 45 couples) characteristics of study patients were comparable with regard to age, the number of treatment failures, the percentage of normal form assessed by MSOME, semen parameters, the total number of oocytes collected, the number of mature oocytes collected, the total number of embryos transferred and the number of high-quality embryos transferred. Similar to the general population, no statistically significant differences $(P>0.05)$ were observed between the two groups with regard to rates of fertilisation (IMSI: $59.1 \pm$ 23.1\%; ICSI: $57.8 \pm 25.2 \%$ ), implantation (IMSI: $11.8 \%$; ICSI: $11.2 \%$ ) and pregnancy/cycle (IMSI: $32.4 \%$; ICSI:
24.4\%). Rates of miscarriage (IMSI: 16.7\%; ICSI: 36.4\%), ongoing pregnancy (IMSI:27\%; ICSI: 15.5\%) and live births (IMSI:27\%; ICSI: 15.5\%) again showed a clear trend towards better outcomes in the IMSI group, although they were not considered statistically significant. These results are summarised in Table 2.

In relation to the subpopulation without male factor (IMSI: 63 couples; ICSI: 55 couples), there were no differences in patient age, the number of treatment failures, the percentage of normal form assessed by MSOME, semen parameters, the total number of oocytes collected, the number of mature oocytes collected, the total number of embryos transferred and the number of high-quality embryos transferred between the two groups. Once again, no differences $(P>0.05)$ were observed in rates of fertilisation (IMSI: $68.1 \pm 24.4 \%$;

Table 1 General study population; comparison between morphologically selected sperm injection (IMSI) and conventional intracytoplasmic sperm injection (ICSI) groups

\begin{tabular}{|c|c|c|c|}
\hline & \multicolumn{3}{|c|}{ Total } \\
\hline & IMSI & ICSI & $P$ \\
\hline Cycles (n) & 100 & 100 & \\
\hline Female age (years) & $36.8 \pm 3.9$ & $36.7 \pm 4.0$ & 0.80 \\
\hline Male age (years) & $39.8 \pm 6.2$ & $40.3 \pm 6.0$ & 0.50 \\
\hline Number of failures & $3.3 \pm 1.7$ & $3.2 \pm 1.6$ & 0.49 \\
\hline \multicolumn{4}{|l|}{ Aetiology (\%) } \\
\hline Male & 29 & 35 & 0.12 \\
\hline Idiopathic & 24 & 17 & \\
\hline Tuboperitoneal & 19 & 14 & \\
\hline Endometriosis & 18 & 18 & \\
\hline Male + endometriosis & 6 & 2 & \\
\hline Tuboperitoenal+ endometriosis & 2 & 6 & \\
\hline Male+tuboperitoneal & 2 & 8 & \\
\hline \multicolumn{4}{|l|}{ MSOME $^{a}$} \\
\hline Normal spermatozoa (\%) & $1.5 \pm 1.8$ & $1.7 \pm 1.8$ & 0.32 \\
\hline \multicolumn{4}{|l|}{ Semen Parameters ${ }^{b}$} \\
\hline Total sperm count $\left(\times 10^{6} / \mathrm{mL}\right)$ & $70.4 \pm 83.4$ & $70.7 \pm 60.4$ & 0.97 \\
\hline Motility (\% spermatozoa) (rapid + slow progression) & $51.8 \pm 19.7$ & $49.6 \pm 23.7$ & 0.55 \\
\hline Vitality (\%) (mean \pm SD) & $60.5 \pm 17.4$ & $56.0 \pm 16.9$ & 0.20 \\
\hline Leukocytes in semen $\left(\times 10^{6}\right)$ (mean \pm SD) & $0.4 \pm 0.6$ & $0.5 \pm 0.4$ & 0.22 \\
\hline \multicolumn{4}{|l|}{ Number of oocytes } \\
\hline Mature (MII) & $7.1 \pm 4.8$ & $6.7 \pm 3.5$ & 0.94 \\
\hline Total & $9.5 \pm 6.2$ & $8.4 \pm 4.6$ & 0.45 \\
\hline Fertilisation rate (\%) & $65.4 \pm 23.5$ & $62 \pm 26.5$ & 0.34 \\
\hline Embryos transfer $(n)$ & $2.7 \pm 1.0$ & $2.7 \pm 1.0$ & 0.44 \\
\hline High-quality embryo transfer $(n)$ & $1.4 \pm 0.5$ & $1.5 \pm 0.5$ & 0.53 \\
\hline Implantation rate (\%) & 13.6 & 9.8 & 0.21 \\
\hline Pregnancy/cycle (\%) & 26 & 19 & 0.73 \\
\hline Miscarriage (\%) & 15.4 & 31.6 & 0.28 \\
\hline Ongoing pregnancy/cycle (\%) & 22 & 13 & 0.13 \\
\hline Live birth/cycle (\%) & 21 & 12 & 0.12 \\
\hline
\end{tabular}

${ }^{a}$ motile sperm organelle morphology examination

b semen quality parameters according to the World Health Organisation[34] 
Table 2 Subpopulations with or without male factor; comparison between morphologically selected sperm injection (IMSI) and conventional intracytoplasmic sperm injection (ICSI) study groups

\begin{tabular}{|c|c|c|c|c|c|c|}
\hline & & male factor & & & ut male factc & \\
\hline & IMSI & ICSI & $P$ & IMSI & ICSI & $P$ \\
\hline Cycles $(n)$ & 37 & 45 & & 63 & 55 & \\
\hline Female age (years) & $36.9 \pm 4.0$ & $35.6 \pm 3.9$ & 0.11 & $36.6 \pm 4.0$ & $37.7 \pm 3.9$ & 0.13 \\
\hline Male age (years) & $40.6 \pm 6.4$ & $41.9 \pm 6.5$ & 0.34 & $39.3 \pm 6.0$ & $39.0 \pm 5.3$ & 0.79 \\
\hline Number of failures & $3.1 \pm 1.6$ & $3.4 \pm 1.9$ & 0.64 & $3.4 \pm 1.7$ & $3.0 \pm 1.3$ & 0.20 \\
\hline Aetiology (\%) & & & & & & \\
\hline Male & 78.4 & 77.8 & 0.08 & & & 0.33 \\
\hline Idiopathic & 0 & 0 & & 38 & 30.9 & \\
\hline Tuboperitoneal & 0 & 0 & & 30.2 & 25.4 & \\
\hline Endometriosis & 0 & 0 & & 28.6 & 32.7 & \\
\hline Male + endometriosis & 16.2 & 4.4 & & 0 & 0 & \\
\hline Tuboperitoenal+ endometriosis & 0 & 0 & & 3.2 & 11 & \\
\hline Male+tuboperitoneal & 5.4 & 17.8 & & 0 & 0 & \\
\hline $\mathrm{MSOME}^{\mathrm{a}}$ & & & & & & \\
\hline Normal spermatozoa (\%) & $0.3 \pm 0.3$ & $0.3 \pm 0.3$ & 0.79 & $2.4 \pm 1.9$ & $2.5 \pm 2.1$ & $>0.99$ \\
\hline Semen Parameters & & & & & & \\
\hline Total sperm count $\left(\times 10^{6} / \mathrm{mL}\right)$ & $14.2 \pm 18.4$ & $11.8 \pm 14.9$ & 0.86 & $95.8 \pm 88.9$ & $98.9 \pm 52.5$ & 0.82 \\
\hline Motility (\% spermatozoa) & $35.5 \pm 14.1$ & $29.6 \pm 15.6$ & 0.12 & $60.9 \pm 16.2$ & $61.1 \pm 10.8$ & 0.94 \\
\hline (rapid+slowprogression) & $49.6 \pm 15.8$ & $47.2 \pm 15.2$ & 0.54 & $65.5 \pm 15.9$ & $70 \pm 6.3$ & 0.56 \\
\hline Vitality (\%) (mean \pm SD) & $0.3 \pm 0.3$ & $0.4 \pm 0.5$ & 0.21 & $0.4 \pm 0.8$ & $0.5 \pm 0.3$ & 0.16 \\
\hline Leukocytes in semen $\left(x 10^{6}\right)$ (mea & & & & & & \\
\hline Number of oocytes & & & & & & \\
\hline Mature (MII) & $8.0 \pm 5.1$ & $7.8 \pm 3.7$ & 0.82 & $6.6 \pm 4.4$ & $5.9 \pm 3.1$ & 0.56 \\
\hline Total & $10.7 \pm 6.8$ & $9.4 \pm 4.6$ & 0.61 & $8.7 \pm 5.7$ & $7.6 \pm 4.4$ & 0.44 \\
\hline Fertilisation rate (\%) & $59.1 \pm 23.1$ & $57.8 \pm 25.2$ & 0.61 & $68.1 \pm 24.4$ & $65.4 \pm 27.4$ & 0.72 \\
\hline Embryos transfer $(n)$ & $2.6 \pm 1.0$ & $2.8 \pm 1.1$ & 0.69 & $2.7 \pm 1.1$ & $2.6 \pm 1.0$ & 0.63 \\
\hline High-quality embryo transfer ( $n$ ) & $1.5 \pm 0.5$ & $1.5 \pm 0.5$ & 0.89 & $1.4 \pm 0.5$ & $1.5 \pm 0.5$ & 0.55 \\
\hline Implantation rate (\%) & 11.8 & 11.2 & 0.88 & 14.6 & 8.6 & 0.16 \\
\hline Pregnancy/cycle (\%) & 32.4 & 24.4 & 0.57 & 22.2 & 14.5 & 0.40 \\
\hline Miscarriage (\%) & 16.7 & 36.4 & 0.54 & 14.3 & 25 & 0.95 \\
\hline Ongoing pregnancy/cycle (\%) & 27 & 15.5 & 0.31 & 19 & 10.9 & 0.33 \\
\hline Live birth/cycle (\%) & 27 & 15.5 & 0.31 & 17.5 & 9.1 & 0.29 \\
\hline
\end{tabular}

a motile sperm organelle morphology examination

${ }^{b}$ semen quality parameters according to the World Health Organisation[34]

ICSI: $65.4 \pm 27.4 \%$ ), implantation (IMSI: $14.6 \%$; ICSI: $8.6 \%$ ) or pregnancy/cycle (IMSI: $22.2 \%$; ICSI: $14.5 \%$ ). As in previous analyses, rates of miscarriage (IMSI: 14.3\%; ICSI: 25\%), ongoing pregnancy (IMSI: 19\%; ICSI: 10.9\%) and live births (IMSI: 17.5\%; ICSI: 9.1\%) displayed a clear trend towards better outcomes in the IMSI group, although they were not statistically significant. These results are summarised in Table 2 .

\section{Discussion}

The aim of ART is to birth a single, healthy baby, and the success of this event depends on many variables. Despite advances in the field of assisted reproduction, implantation rates have not improved significantly since the ICSI technique was established in 1992 [36]. It is well known that implantation rates are related to endometrial receptivity, cytokines, and growth factors secreted by ovarian stimulation [37-39]. Poor embryo quality is another important factor that negatively affects implantation rates [40]. Choosing sperm using MSOME parameters appears to provide better rates of fertilisation and embryo quality $[11,21]$, which also translates to better conditions during the implantation process. It is well established that the capacity of human sperm to fertilise an oocyte and produce an embryo with high potential of implantation and development depends, among other factors, on DNA integrity $[15,16]$. Observations from our group have shown that the presence of large nuclear vacuoles (LNV), which are sperm-specific alterations observed at high magnification, may be related to DNA fragmentation and 
denaturation [15]. Vanderzwalmen et al. [24] also reported a negative impact of the presence of one large nuclear vacuole or several small vacuoles in the head of spermatozoa on the competence of embryos to develop to the blastocyst stage. Moreover, Wilding et al. [20] had suggested that a correlation exists between LNV and DNA fragmentation. Even if percentages of fertilisation and embryo development are comparable to those of conventional ICSI, embryos obtained from spermatozoa analysed by MSOME showed higher quality, and more overall pregnancies were achieved. On the other hand, recent investigations have related LNV to the absence of an acrosome reaction, which could explain the hampering of embryo development when a spermatozoon with this characteristic is chosen for microinjection [41]. Gopalkrishnan et al. [42] found that the chromatin of spermatozoa from men whose partners presented with early pregnancy loss was often compact or partially compact with irregular nuclear borders and large vacuoles.

Currently, ICSI is performed after morphological selection of spermatozoa at $200 \times$ to $400 \times$ magnification. In this magnification range, spermatozoa carrying defects of the head, neck or tail can be detected, but nuclear vacuoles cannot. Therefore, observation at a magnification of $6000 \times$ to $12,500 \times$ when selecting spermatozoa appears to be better by simply allowing precise identification of vacuoles $[6,7,15,16,20,23,24]$. Nevertheless, in couples with repeated implantation failures, sperm selection at high magnification does not appear to exert much significant influence. Fertilisation rates were similar between the IMSI and ICSI groups, a result not influenced by the presence or absence of male factor, and confirms other prior findings that also did not detect any significant differences in fertilisation rates $[5,11,12,23]$. Regarding clinical results, there was again no significant influence of sperm selection at high magnification both in the general population and in subgroups with or without male factor. However, there was a significant and clear trend for better results in the IMSI group, particularly for rates of ongoing pregnancy, live births (both nearly doubled) and miscarriage ( $\approx 50 \%$ reduced). Although not statistically significant (likely due to sample size), these results can be considered clinically relevant. Given that apparently normal spermatozoa visualized in the low magnification range in conventional ICSI may possess hidden anomalies that could be detected at higher magnification with MSOME, the use of sperm selected by IMSI can produce embryos with higher developmental capacity. This would exclude using sperm with higher probabilities of DNA fragmentation and denaturation.

This trend is in agreement with several published studies. In addition, other investigations reported that IMSI is able to increase pregnancy rates and reduce miscarriage rates in assisted reproduction cycles $[5,11,14,20,23,26]$. In a prospective randomized study comparing the clinical outcome of 87 IMSI cycles with 81 conventional ICSI cycles, Balaban et al. [25] reported that IMSI did not provide any significant improvement in the clinical outcome compared to ICSI. However, there were trends for higher rates of implantation (28.9\% versus $19.5 \%$ ), clinical pregnancy $(54.0 \%$ versus $44.4 \%)$ and live births (43.7\% versus $38.3 \%)$ in the IMSI group. On the other hand, in this same study [25], severe male factor patients were found to benefit from the IMSI procedure, as significantly higher implantation rates were observed compared to their counterparts in the ICSI group.

In conclusion, the results obtained in this investigation suggest that IMSI does not appear to provide a significant improvement in clinical outcome compared to ICSI, at least in couples with repeated implantation failures after conventional ICSI. However, it should be noted that there were clear trends for lower rates of miscarriage $(\approx 50 \%$ reduced) and higher ongoing pregnancy and live births (nearly doubled) in the IMSI group. Thus, using morphologically-selected spermatozoa with IMSI would benefit those with poor reproductive prognosis; however, further confirmation in addition to randomized large-scale trials are still needed.

\section{Acknowledgements}

The authors wish to thank the American Journal Experts http://www. journalexperts.com for revising the English text.

\section{Author details}

'Department of Gynecology and Obstetrics, Botucatu Medical School Sao Paulo State University - UNESP, Botucatu, Brazil. ${ }^{2}$ Paulista Centre for Diagnosis, Research and Training, Ribeirao Preto - SP, Brazil. ${ }^{3}$ Centre for Human Reproduction, Prof. Franco Jr., Ribeirao Preto, Brazil. ${ }^{4}$ Postgraduate Fellow Department of Gynecology and Obstetrics, Botucatu Medical School Sao Paulo State University - UNESP, Brazil.

\section{Authors' contributions}

JBAO designed and coordinated the study. All authors were responsible for data collection, analysis, and interpretation presented in the manuscript. JBAO, MC, RLRB and JGF performed the statistical analyses and wrote the manuscript; JGF reviewed the manuscript. All authors read and approved the final manuscript.

\section{Conflicts of Interests}

The authors declare that they have no competing interests.

Received: 8 March 2011 Accepted: 22 July 2011 Published: 22 July 2011

\section{References}

1. Seli E, Vergouw CG, Morita H, Botros L, Roos P, Lambalk CB, Yamashita N, Kato O, Sakkas D: Noninvasive metabolomic profiling as an adjunct to morphology for noninvasive embryo assessment in women undergoing single embryo transfer. Fertil Steril 2010, 94:535-542.

2. Tasdemir I, Tasdemir M, Tavukcuoglu S, Kahraman S, Biberoglu K: Effect of abnormal sperm head morphology on the outcome of intracytoplasmic sperm injection in humans. Hum Reprod 1997, 12:1214-1217.

3. De Vos A, Van De Velde H, Joris H, Verheyen G, Devroey P, Van Steirteghem A: Influence of individual sperm morphology on fertilization, embryo morphology, and pregnancy outcome of intracytoplasmic sperm injection. Fertil Steril 2003, 79:42-48. 
4. Gvakharia M, Beri ZL, Adamson GD: Correlation Between Sperm Morphology by Kruger's Strict Criteria and the Outcome of ICSI in IVF Cycles. Fertil Steril 2005, 84:S459.

5. Bartoov B, Berkovitz A, Eltes F, Kogosovsky A, Yagoda A, Lederman H, Artzi S, Gross M, Barak Y: Pregnancy rates are higher with intracytoplasmic morphologically selected sperm injection than with conventional intracytoplasmic injection. Fertil Steril 2003, 80:1413-1419.

6. Berkovitz A, Eltes F, Yaari S, Katz N, Barr I, Fishman A, Bartoov B: The morphological normalcy of the sperm nucleus and pregnancy rate of intracytoplasmic injection with morphologically selected sperm. Hum Reprod 2005, 20:185-190.

7. Berkovitz A, Eltes F, Ellenbogen A, Peer S, Feldberg D, Bartoov B: Does the presence of nuclear vacuoles in human sperm selected for ICSI affect pregnancy outcome? Hum Reprod 2006, 21:1787-1790.

8. Tesarik J, Mendoza C, Greco E: Paternal effects acting during the first cell cycle of human preimplantation development after ICSI. Hum Reprod 2002, 17:184-189.

9. Tesarik J, Greco E, Mendoza C: Late, but not early, paternal effect on human embryo development is related to sperm DNA fragmentation. Hum Reprod 2004, 19:611-615.

10. Tesarik J: Paternal effects on cell division in the human preimplantation embryo. Reprod Biomed Online 2005, 10:370-375.

11. Hazout A, Dumont-Hassan M, Junca AM, Cohen Bacrie P, Tesarik J: Highmagnification ICSI overcomes paternal effect resistant to conventional ICSI. Reprod Biomed Online 2006, 12:19-25.

12. Bartoov B, Berkovitz A, Eltes F, Kogosowski A, Menezo Y, Barak Y: Real-time fine morphology of motile human sperm cells is associated with IVF-ICSI outcome. J Androl 2002, 23:1-8.

13. Antinori M, Licata E, Dani G, Cerusico F, Versaci C, d'Angelo D, Antinori S: Intracytoplasmic morphologically selected sperm injection: a prospective randomized trial. Reprod Biomed Online 2008, 16:835-841.

14. Nadalini M, Tarozzi $N$, Distratis $V$, Scaravelli G, Borini A: Impact of intracytoplasmic morphologically selected sperm injection on assisted reproduction outcome: a review. Reprod Biomed Online 2009, 19(Suppl 3):45-55.

15. Franco JG Jr, Baruffi RL, Mauri AL, Petersen CG, Oliveira JB, Vagnini L: Significance of large nuclear vacuoles in human spermatozoa: implications for ICSI. Reprod Biomed Online 2008, 17:42-45.

16. Garolla A, Fortini D, Menegazzo M, De Toni L, Nicoletti V, Moretti A, Selice R, Engl B, Foresta C: High-power microscopy for selecting spermatozoa for ICSI by physiological status. Reprod Biomed Online 2008, 17:610-616.

17. Oliveira JB, Massaro FC, Baruffi RL, Mauri AL, Petersen CG, Silva LF, Vagnini LD, Franco JG Jr: Correlation between semen analysis by motile sperm organelle morphology examination and sperm DNA damage. Fertil Steril 2010, 94:1937-1940.

18. Perdrix A, Travers A, Chelli MH, Escalier D, Do Rego JL, Milazzo JP, MoussetSimeon N, Mace B, Rives N: Assessment of acrosome and nuclear abnormalities in human spermatozoa with large vacuoles. Hum Reprod 2011, 26:47-58

19. Boitrelle F, Ferfouri F, Petit JM, Segretain D, Tourain C, Bergere M, Bailly M, Vialard F, Albert M, Selva J: Large human sperm vacuoles observed in motile spermatozoa under high magnification: nuclear thumbprints linked to failure of chromatin condensation. Hum Reprod 2011, 26:1650-1658.

20. Wilding M, Coppola G, di Matteo L, Palagiano A, Fusco E, Dale B: Intracytoplasmic injection of morphologically selected spermatozoa (IMSI) improves outcome after assisted reproduction by deselecting physiologically poor quality spermatozoa. J Assist Reprod Genet 2011, 28:253-262.

21. Yazbeck C, Delaroche L, Jacquesson L, A JP, J S, N R: Intracytoplasmic morphologically selected sperm injection (IMSI): is it a good choice after two or more IVF or ICSI failures? Fertil Steril 2008, 90:5416.

22. Junca AM, Cohen-Bacrie $P$, Hazout A: Improvement of fertilization and pregnancy rate after intracytoplasmic fine morphologically selected sperm injection. Fertil Steril 2004, 82:S173.

23. Berkovitz A, Eltes F, Lederman H, Peer S, Ellenbogen A, Feldberg B, Bartoov B: How to improve IVF-ICSI outcome by sperm selection. Reprod Biomed Online 2006, 12:634-638.

24. Vanderzwalmen $P$, Hiemer $A$, Rubner $P$, Bach $M$, Neyer $A$, Stecher $A$, Uher $P$, Zintz M, Lejeune B, Vanderzwalmen $S$, et al: Blastocyst development after sperm selection at high magnification is associated with size and number of nuclear vacuoles. Reprod Biomed Online 2008, 17:617-627.
25. Balaban B, Yakin K, Alatas C, Oktem O, Isiklar A, Urman B: Clinical outcome of intracytoplasmic injection of spermatozoa morphologically selected under high magnification: a prospective randomized study. Reprod Biomed Online 2011.

26. Souza Setti A, Ferreira RC, Paes de AlmeidaFerreira Braga D, de Cassia Savio Figueira R, laconelli A Jr, Borges E Jr: Intracytoplasmic sperm injection outcome versus intracytoplasmic morphologically selected sperm injection outcome: a meta-analysis. Reprod Biomed Online 2010, 21:450-455.

27. Tasaka A, Doshida M, Sato Y, Kyoya T, Nakajo Y, Kyono K: Outcome of IMSI (intracytoplasmic morphologically selected sperm injection) in patients with repeated ICSI failures. Fertil Steril 2009, , Supplement: S76.

28. Berkovitz A, Eltes F, Paul M, Adrian E, Bartoov B: The chance of having a healthy normal child following intracytoplasmic morphologicallyselected sperm injection (IMSI) treatment is higher compared to conventional IVF-ICSI treatment. Fertil Steril 2007, 88:S20.

29. Ai L, Liu SY, Huang J, Chen SW, Liu J, Zhong Y: [Intracytoplasmic morphologically selected sperm injection of testicular sperm: clinical outcome in azoospermia patients]. Zhonghua Nan Ke Xue 2010, 16:826-829.

30. Oliveira JB, Massaro FC, Mauri AL, Petersen CG, Nicoletti AP, Baruffi RL, Franco JG Jr: Motile sperm organelle morphology examination is stricter than Tygerberg criteria. Reprod Biomed Online 2009, 18:320-326.

31. Mauri AL, Petersen CG, Oliveira JB, Massaro FC, Baruffi RL, Franco JG Jr: Comparison of day 2 embryo quality after conventional ICSI versus intracytoplasmic morphologically selected sperm injection (IMSI) using sibling oocytes. Eur J Obstet Gynecol Reprod Biol 2010, 150:42-46.

32. Holte J, Berglund L, Milton K, Garello C, Gennarelli G, Revelli A, Bergh T: Construction of an evidence-based integrated morphology cleavage embryo score for implantation potential of embryos scored and transferred on day 2 after oocyte retrieval. Hum Reprod 2007, 22:548-557.

33. Saldeen P, Sundstrom P: Nuclear status of four-cell preembryos predicts implantation potential in in vitro fertilization treatment cycles. Fertil Steril 2005, 84:584-589.

34. World Health Organization: WHO laboratory manual for the examination of human semen and sperm-cervical mucus interaction. 4 edition. Cambridge, UK; New York, NY: Published on behalf of the World Health Organization by Cambridge University Press; 1999.

35. ASTM: Standart E1951-02 Physical and mechanical testing standards. West Conshohocken, PA 2007.[http://www.astm.org].

36. Palermo G, Joris $H$, Devroey $P$, Van Steirteghem AC: Pregnancies after intracytoplasmic injection of single spermatozoon into an oocyte. Lancet 1992, 340:17-18.

37. Horne AW, White JO, Lalani EN: The endometrium and embryo implantation. A receptive endometrium depends on more than hormonal influences. BMJ 2000, 321:1301-1302.

38. Bonetti TC, Salomao R, Brunialti M, Braga DP, Borges E Jr, Silva ID: Cytokine and hormonal profile in serum samples of patients undergoing controlled ovarian stimulation: interleukin-1 beta predicts ongoing pregnancy. Hum Reprod 2010, 25:2101-2106.

39. Joo BS, Park SH, An BM, Kim KS, Moon SE, Moon HS: Serum estradiol levels during controlled ovarian hyperstimulation influence the pregnancy outcome of in vitro fertilization in a concentration-dependent manner. Fertil Steril 2010, 93:442-446.

40. Urman B, Yakin K, Balaban B: Recurrent implantation failure in assisted reproduction: how to counsel and manage. A. General considerations and treatment options that may benefit the couple. Reprod Biomed Online 2005, 11:371-381.

41. Kacem O, Sifer C, Barraud-Lange V, Ducot B, De Ziegler D, Poirot C, Wolf J: Sperm nuclear vacuoles, as assessed by motile sperm organellar morphological examination, are mostly of acrosomal origin. Reprod Biomed Online 2010, 20:132-137.

42. Gopalkrishnan K, Padwal V, Meherji PK, Gokral JS, Shah R, Juneja HS: Poor quality of sperm as it affects repeated early pregnancy loss. Arch Androl 2000, 45:111-117.

doi:10.1186/1477-7827-9-99

Cite this article as: Oliveira et al:: Pregnancy outcomes in women with repeated implantation failures after intracytoplasmic morphologically selected sperm injection (IMSI). Reproductive Biology and Endocrinology 2011 9:99. 